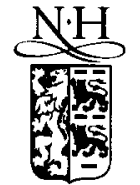

ELSEVIER

\title{
Studies of the lattice Anderson model
}

\author{
Y.C. Cheng ${ }^{a, *}$, J.W. Chen ${ }^{a}$, H.Q. Lin ${ }^{\mathrm{a}, \mathrm{b}}$ \\ a Department of Physics, National Taiwan University, Taipei, 106 Taiwan \\ b Department of Physics, University of Illinois at Urbana - Champaign, 1110 West Green Street, Urbana, IL 61801, USA
}

\begin{abstract}
By combining the exact diagonalization technique and perturbation theory, we study the symmetric lattice Anderson model in one dimension. The model is characterized by the Coulomb repulsion $U$ and the energy gap $\Delta=\sqrt{t^{2}+V^{2}}-t$, where $t$ is the hopping integral for the conduction electrons and $V$ is the hybridization matrix. We obtain the ground state energy per site and the spin-spin correlations by carrying out perturbation theory to fourth order in $V$. Comparisons with results obtained from exact diagonalization calculations were made and we pointed out regions where perturbative expansions could be invalid.
\end{abstract}

In this paper, we report our recent studies of the one-dimensional lattice Anderson model. This model has been studied extensively in recent years in connection with the valence fluctuation problems and an extensive literature exists [1-3]. Several approximation techniques [1-5] have been applied to deal with the many-body aspects of this model including renormalization group analysis, the Gutzwiller variational approach, the large-orbital-degeneracy $1 / N$ expansion, the slave boson mean-field approach, and the second order perturbation theory [6]. Numerical calculations have been carried out [7-16]. The model is defined by the Hamiltonian

$$
\begin{aligned}
H= & -t \sum_{i \sigma}\left(c_{i \sigma}^{\dagger} c_{i+1 \sigma}+\text { h.c. }\right)+E_{\mathrm{f}} \sum_{i \sigma} n_{f i \sigma} \\
& +U \sum_{i} n_{f i \uparrow} n_{f i \downarrow}+V \sum_{i \sigma}\left(c_{i \sigma}^{\dagger} f_{i \sigma}+\text { h.c. }\right),
\end{aligned}
$$

where $c_{i \sigma}^{\dagger}\left(c_{i \sigma}\right)$ and $f_{i \sigma}^{\dagger}\left(f_{i \sigma}\right)$ are, respectively, the creation (annihilation) operators for electrons in the $\mathrm{d}$ - and the f-orbitals on site $i$ with spin $\sigma$, and $n_{f i \sigma}=f_{i \sigma}^{\dagger} f_{i \sigma}$. The d electrons hop with an amplitude $t$, thus forming a conduction band $e_{k}=-2 t \cos k$. The $\mathrm{f}$ electrons hybridize with the $\mathbf{d}$ electrons with an amplitude $V$, and have usual Hubbard interaction $U$ between spin up and down electrons on the same site. The site energy $E_{\mathrm{f}}$ for an f electron defines the relative position of the $f$ states with respect to the Fermi energy of the $d$ electrons. An important special case is the so called symmetric Anderson model in which $E_{\mathrm{f}}=-U / 2$.

\footnotetext{
${ }^{*}$ Corresponding author. Fax: +886-2-363-9984; email: yc cheng@phys.ntu.edu.tw.
}

When $U=0$, one can diagonalize Eq. (1) easily. A two-band structure is obtained with band energies

$E_{k}^{ \pm}=\frac{1}{2}\left[e_{k}+E_{\mathrm{f}} \pm \sqrt{\left(e_{k}-E_{\mathrm{f}}\right)^{2}+4 V^{2}}\right]$

A characteristic quantity in the Anderson lattice model is the energy gap $\Delta$ which separates the two bands, where $\Delta=\frac{1}{2}\left(E_{k=0}^{+}-E_{k=\pi}^{-}\right)=\sqrt{t^{2}+V^{2}}-t$. The magnitude of the ratio $U / \Delta$ defines the weak and the strong coupling regions.

We have used perturbation theory and exact diagonalization methods to study the ground state properties of the model Eq. (1). Exact diagonalization studies were performed on finite lattices with site number $N=2,4,6$ and 8. Lack of computer memory prevented us to go beyond $N=8$. However, by studying the lattice-size dependence of data, we noticed that they oscillate between lattice size $N=4 n$ and $N=4 n+2$. This can be shown analytically for a 1D tight binding band [11]. For the interacting case, we can show it numerically. We also found that data obtained from different boundary conditions (such as antiperiodic or open boundary) also possess such nice feature. Thus, we can use data obtained from $N=4 n$ and $4 n+2$ to set up the upper and the lower bounds for the results of infinite systems. We have also tried other extrapolation schemes by assuming asymptotic forms of calculated quantities, and we found that they converge very well so we were able to obtain results for infinite systems with reasonable accuracy.

Analytically, there are two limits where one can perform perturbation calculations. One is the weak coupling limit, and the other is the strong coupling limit. In the 
strong coupling limit, where $U / \Delta$ is large, the unperturbated Hamiltonian for the symmetric case $\left(E_{\mathrm{f}}=-U / 2\right)$ is

$$
\begin{aligned}
H_{0}= & \sum_{k} e_{k} c_{k \sigma}^{\dagger} c_{k \sigma}-\frac{U}{2} \sum_{i}\left(n_{f i \uparrow}-n_{f i \downarrow}\right)^{2} \\
& +h_{\mathrm{s}} \sum_{i}(-1)^{i}\left(n_{f i \uparrow}-n_{f i \downarrow}\right),
\end{aligned}
$$

where we have added a staggered magnetic field $h_{\mathrm{s}}$ acting on the impurity sites so the ground state of $H_{0}$ is nondegenerate. This is reasonable because in the strong coupling limit the system is expected to form short-range local moments antiferromagnetically. The limit $h_{\mathrm{s}} \rightarrow 0$ will be taken at the end of calculations. The pertubated Hamiltonian is just the hybridization part of Eq. (1),

$H_{1}=\frac{V}{\sqrt{N}} \sum_{i \sigma k}\left(\mathrm{e}^{\mathrm{i} k r_{i}} c_{k \sigma}^{\dagger} f_{i \sigma}+\right.$ h.c. $)$.

We have calculated the ground state energy per site to fourth order in $V$, the result is

$$
\begin{aligned}
& E(U)=E_{0}(U)+E_{2}(U)+E_{4}(U), \\
& E_{0}(U)=-\frac{U}{2}-h_{\mathrm{s}}+\frac{2}{N} \sum_{k} e_{k} f\left(e_{k}\right), \\
& E_{2}(U)=-\frac{2 V^{2}}{N} \sum_{k} \frac{1-f\left(e_{k}\right)}{D_{+}(k)}, \\
& E_{4}(U) \\
& \quad-\left(\frac{V^{4}}{2 N^{2}}\right)\left[\sum_{k}\left(\frac{f\left(e_{k}\right)}{\left[D_{-}(k)\right]^{3}}+\frac{1-f\left(e_{k}\right)}{\left[D_{+}(k)\right]^{3}}\right)\right. \\
& \quad \times\left(\frac{1-f\left(e_{k_{1}, k_{2}}\right)}{D_{-}\left(k_{1}\right)}\right)\left(\frac{f\left(e_{k_{1}}\right)}{D_{-}\left(k_{1}\right)}+\frac{1-f\left(e_{k_{2}}\right)}{D_{+}\left(k_{2}\right)}\right) \\
& D_{+}\left(k_{2}\right)-D_{+}\left(k_{1}\right)
\end{aligned}
$$

where $f\left(e_{k}\right)$ is the zero temperature Fermi factor and $D \pm(k)=U / 2+h_{\mathrm{s}} \pm e_{k}$. (In Eq. (5), $E_{1}(U)=E_{3}(U)=0$ as any odd power term in $V$ vanishes.) Note that the term $U / 2$ is just a constant coming from the chemical potential, and the second summation in Eq. (8) is of order $1 / U^{2}$, not $1 / U^{3}$ as one might naively think. Thus, the convergence in the strong coupling region could be slower than in the weak coupling region in which the ground state energy per site is expanded in a power series of $U[6,7]$.

We have performed exact diagonalization and perturbation calculations for the symmetric case with $t=1.0$, $V=0.5$ (hence $\Delta=0.12$ ), in the $\frac{1}{2}$-filled sector. The ground state energy $E(U)$, local moments $m_{f z}^{2}$, and the effective hybridization $\left\langle c_{i \sigma}^{\dagger} f_{i \sigma}+\right.$ h.c. $\rangle$, were calculated as functions of $U / t$ varying from 0 to 4 , i.e, from the weak

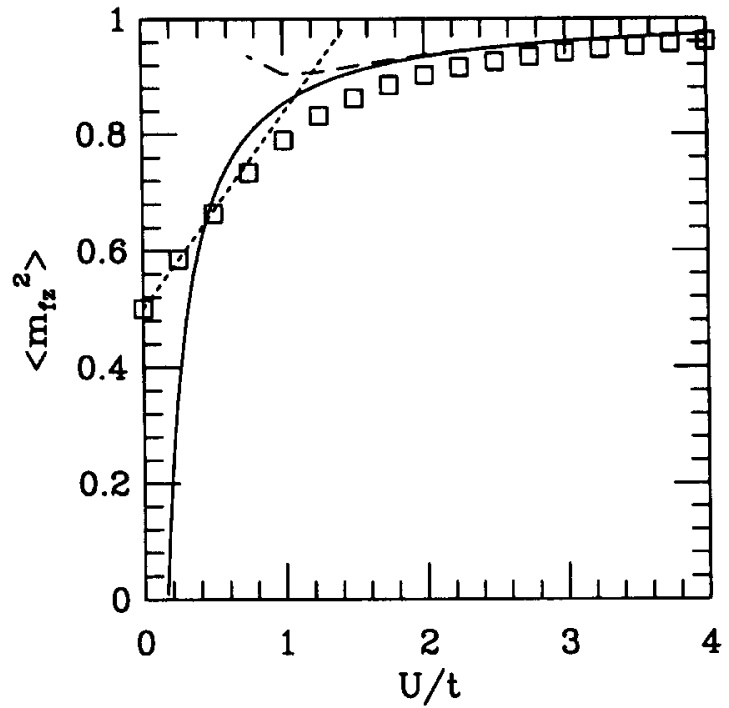

Fig. 1. Square of the $f$ orbital local moment as a function of $U$ for the one-dimensional symmetric Anderson lattice model with $t=1$, $V=0.5$. The dashed line is the weak coupling perturbation result, the solid and the dot-dashed lines are, respectively, the second and the fourth order strong coupling perturbation results. The squares are the exact diagonalization results and the uncertainty in the infinite-system extrapolation is within the size of the symbol.

to the strong coupling limits. Due to space restrictions, we only show the results of local moments, defined as

$m_{f z}^{2}=\left\langle\left(n_{f \uparrow}-n_{f \downarrow}\right)^{2}\right\rangle=-2 \partial E / \partial U$,

in Fig. 1. By comparing with exact diagonalization results, it is evident that the weak coupling perturbation is invalid for $U / \Delta>8$ ( $m_{f z}^{2}$ can never exceed 1$)$, and the strong coupling perturbation gives bad answers for $U / \Delta<16$. Fig. 1 also shows that the 4th order corrections seem to give better answers for large $U$ as we hoped. However, the 2nd and the 4th order results start to deviate from each other as $U / t<2$, reconfirming that the strong coupling expansion becomes inadequate for $U / \Delta<16$. This may cause some doubt on the applicability of the slave boson technique to this model [5], where a small value of $U_{\mathrm{c}}$ was found, but expansions for the $s-d$ model could lead to a different result. In the intermediate coupling region, exact diagonalization results connect the two limits smoothly, and we hope that the perturbative calculations could be improved by carrying out to higher orders.

Acknowledgements: H.Q. Lin is grateful to H. Chen for computational assistance and he thanks J. Callaway for useful discussions. This research was supported by the National Science Council of the Republic of China under contract No. NSC83-0212-M-002-074.

\section{References}

[1] P. Fulde, J. Keller and G. Zwicknagel, in: Solid State Physics, Vol. 41, eds. H. Ehrenreich and D. Turnbull (Academic, New York, 1988) p.1 and references therein.

[2] D.M. Newns and N. Read, Adv. Phys. 36 (1987) 799. 
[3] N.E. Bickers, Rev. Mod. Phys. 59 (1987) 845.

[4] A.J. Mills and P.A. Lee, Phys. Rev. B 35 (1987) 3394.

[5] T.M. Hong and G.A. Gehring, J. Magn. Magn. Mater. 108 (1992) 93; M.F. Yang, S.J. Sun and T.M. Hong, Phys. Rev. B 48 (1993) 16123; ibid. 48 (1993) 16127.

[6] K. Yamada and K. Yosida, in: Procs. 3rd Taniguchi Symp., Mount Fuji, Japan, 1980, ed. T. Moriya (Springer-Verlag, Berlin, 1981) p. 210; K. Yamada, Prog. Theor. Phys. 53 (1975) 970.

[7] R. Blankenbecler, J.R. Fulco, W. Gill and D.J. Scalapino, Phys. Rev. Lett. 58 (1987) 411.

[8] J.E. Gubernatis, J.E. Hirsch and D.J. Scalapino, Phys. Rev. B 35 (1987) 8478; ibid. 36 (1987) 394.

[9] R.M. Fye, J.E. Hirsch and D.J. Scalapino, Phys. Rev. B 35 (1987) 4901; ibid. 44 (1991) 7486.
[10] J. Callaway, D.P. Chen, D.G. Kanhere and P.K. Misra, Phys. Rev. B 38 (1988) 2583; Y. Zhang and J. Callaway, ibid. 38 (1988) 641; J. Callaway, J.W. Kim, L. Tan and H.Q. Lin, ibid. 48 (1993) 11545; J. Callaway, D.G. Kanhere and H.Q. Lin, J. Appl. Phys. 73 (1993) 5406.

[11] H.Q. Lin, H. Chen and J. Callaway, J. Appl. Phys. 75 (1994) 7041.

[12] A. Reich and L.M. Falicov, Phys. Rev. B 34 (1986) 6752.

[13] J.A. White, Phys. Rev. B 46 (1992) 13905.

[14] P. Santini, L. Andreani and H. Beck, Phys. Rev. B 47 (1993) 1130.

[15] K. Ueda, J. Phys. Soc. Jpn. 58 (1989) 3465; K. Yamamoto and K. Ueda, ibid. 59 (1990) 3284.

[16] R. Jullien and R.M. Martin, Phys. Rev. B 26 (1982) 6173. 